\title{
Cefepime monotherapy for the empirical treatment of fever in granulocytopenic cancer patients
}

\author{
P. Eggimann ${ }^{a}$, M. P. Glauser ${ }^{a}$, M. Aoun ${ }^{b}$, F. Meunier ${ }^{b}$ and T. Calandra ${ }^{a}$ \\ ${ }^{a}$ Division of Infectious Diseases, Department of Internal Medicine, Centre Hospitalier \\ Universitaire, Vaudois, CH-1011, Lausanne, Switzerland; ${ }^{b}$ Infectious Diseases Unit, \\ Department of Medicine, Institut Jules Bordet, Rue Héger, B-1000 Brussels, Belgium
}

\begin{abstract}
In a pilot study, we evaluated the efficacy and the safety of cefepime, a new cephalosporin with extended-spectrum activity against both Gram-positive and Gram-negative bacteria, as empirical monotherapy for 108 febrile episodes in 84 granulocytopenic cancer patients. Cefepime ( $2 \mathrm{~g}$ tds) was given for a minimum of 7 days or until resolution of infection. Of the 108 episodes, 91 were evaluable. Microbiologically documented infections occurred in 25 patients $(27 \%)(18$ Gram-positive, 7 Gram-negative), of whom 18 had bacteraemia. Infection was clinically documented in 47 patients $(52 \%)$ and fever was unexplained in $19(21 \%)$.

Overall, $71 \%(65 / 91)$ of the infections resolved. Response rates were $86 \%(6 / 7)$ for Gram-negative infections, $44 \%(8 / 18)$ for Gram-positive infections $(57 \%$ for cefepime-susceptible Gram-positive bacteria), 77\% (36/47) for clinically documented infections and $79 \%(15 / 19)$ for unexplained fevers. Of the 26 patients $(29 \%)$ whose primary infections did not improve with cefepime monotherapy, 23 responded after the addition of other antibiotics. Sixteen patients (18\%) developed secondary infections of which 13 were microbiologically documented; Gram-positive bacteria were isolated from seven patients, Gram-negative bacteria from two, fungi from three and a virus from one. Adverse effects were mild and did not require premature discontinuation of therapy except for one patient who developed an immediate allergic reaction after the first dose of cefepime from which he recovered fully. The survival rate after resolution of granulocytopenia was $96 \%$; three patients died of primary bacterial infection and one from secondary disseminated candidiasis.

In this pilot study, cefepime monotherapy appeared safe and effective as empirical therapy for fever in cancer patients with granulocytopenia. Whether cefepime is superior to other advanced-generation cephalosporins for the treatment of Gram-postive infections will require evaluation in a larger comparative study.
\end{abstract}

\section{Introduction}

For many years, combinations of $\beta$-lactam antibiotics and aminoglycosides have been standard therapy for suspected infections of bacterial aetiology in cancer patients with granulocytopenia (Young, 1989; Calandra \& Cometta, 1991). Recent studies have shown that monotherapy with ceftazidime (Fainstein et al., 1983; Pizzo et al., 1986; Deresinski, De Pauw \& Feld, 1991) or imipenem-cilastatin (Winston et al., 1991; Rolston et al., 1992) was as effective overall as combination therapy for the empirical treatment of such infections. However, combination therapy may still be the treatment

*Corresponding author. 
of choice in high-risk patients, such as those with Gram-negative bacteraemia and persistent or profound granulocytopenia (EORTC, 1987).

Currently, Gram-positive bacteria, especially coagulase-negative staphylococc and viridans streptococci, have replaced Gram-negative bacilli as the predominant pathogens in the neutropenic host. Third-generation cephalosporins such as ceftazidime are highly active against Gram-negative bacteria but are suboptimal treatment for infections caused by Gram-positive bacteria. Also, the extended-spectrum penicillins are not active against $\beta$-lactamase-producing Gram-positive bacteria while, in general, $\beta$-lactams are inactive against methicillin-resistant staphylococci. Therefore, vancomycin or teicoplanin have been used empirically to improve the cover against Gram-positive bacteria. However, four studies have now shown that glycopeptide antibiotics need not be part of the initial antibiotic regimen in these patients (Rubin et al., 1988; EORTC, 1991b; Novakova, Donnelly \& De Pauw, 1991; Ramphal et al., 1992). Their use should be limited, therefore, to patients with suspected or confirmed methicillin-resistant staphylococcal infections or with Gram-positive infections not responding to specific therapy. The indiscriminate use of glycopeptide antibiotics might otherwise result in the emergence of resistant staphylococci or enterococci, which would be highly undesirable. Thus, other therapeutic options should be aimed at improving the outcome of Gram-positive infections.

Cefepime is a new injectable cephalosporin with excellent activity against aerobic Gram-negative and Gram-positive bacteria (Kessler et al., 1985; Dornbusch, Mortsell \& Goransson, 1990). MICs of cefepime and ceftazidime are similar for Gram-negative bacteria including Pseudomonas aeruginosa. Against methicillinsusceptible staphylococci and viridans streptococci, the MICs of cefepime are ten times lower than those of ceftazidime and similar to those of vancomycin (Kessler et al., 1985). Against 90 Gram-positive bacteria isolated from blood cultures at the Centre Hospitalier Universitaire Vaudois (Lausanne, Switzerland), the $\mathrm{MIC}_{90} \mathrm{~s}$ of cefepime were $4 \mathrm{mg} / \mathrm{L}$ for methicillin-susceptible Staphylococcus aureus $(n=25), 2 \mathrm{mg} / \mathrm{L}$ for methicillin-susceptible coagulase-negative staphylococci $(n=19), 1 \mathrm{mg} / \mathrm{L}$ for viridans streptococci $(n=24)$ and $0 \cdot 12 \mathrm{mg} / \mathrm{L}$ for $\beta$-haemolytic streptococci $(n=22)$. The clinical efficacy of cefepime has been evaluated in more than 500 mostly non-neutropenic patients (data on file, Bristol-Myers Squibb \& Co., Princeton, NJ, USA). Clinical and bacteriological responses were respectively $95 \%$ and $88 \%$ for urinary tract infections, $95 \%$ and $82 \%$ for skin and soft tissue infections, and $94 \%$ and $88 \%$ for lower respiratory tract infections (data on file, Bristol-Myers Squibb \& Co., Princeton, NJ, USA; Oster et al., 1990).

The purpose of this prospective pilot study was to investigate the efficacy and tolerance of cefepime as empirical monotherapy for febrile episodes in granulocytopenic cancer patients. Clinical efficacy was evaluated according to three different methods of assessment.

\section{Material and methods}

\section{Patient selection}

Patients were eligible for inclusion if they had cancer, granulocytopenia (defined as an absolute granulocyte count of $<1.0 \times 10^{9} / \mathrm{L}$ ) and fever (defined as a temperature of $>38.0^{\circ} \mathrm{C}$ on one occasion). Patients were not eligible if they were younger than 16 years of age, pregnant or nursing, had known hypersensitivity to $\beta$-lactam antibiotics, 
had a serum creatinine $>300 \mu \mathrm{mol} / \mathrm{L}$, had already been entered into the study during the same episode of neutropenia or had received parenteral antibiotics $<4$ days before randomization. Patients receiving oral antibiotics for the prevention of bacterial infection were eligible, provided the prophylaxis was stopped when iv antibiotics were initiated.

The protocol was approved by the Ethics Committee of the two participating institutions and the study was conducted in accordance with the Recommendations Guiding Medical Doctors in Biomedical Research Involving Human Subjects (Helsinki Declaration). All patients gave written or oral informed consent.

\section{Initial and follow-up patient evaluation}

At study entry and daily thereafter, a complete history was obtained from all patients and they underwent a physical examination. The laboratory tests performed at randomization and at least twice-weekly during the study included determinations of haemoglobin, haematocrit, WBC and differential count, platelet count, prothrombin time. partial thromboplastin time, sodium, potassium, blood urea, serum creatinine, bilirubin, serum aspartate aminotransferase, serum alanine aminotransferase, alkaline phosphatase and urinalysis. A chest X-ray, blood cultures (two sets, each from separate venepunctures performed at $30 \mathrm{~min}$ intervals), urine specimens and specimens from any clinically suspected site of infection were also obtained from all patients. In patients with bacteraemia or with persistent fever, blood cultures were repeated daily until resolution of infection.

\section{Participating centres}

The study was conducted at the Centre Hospitalier Universitaire Vaudois, Lausanne, Switzerland (68 patients enrolled) and at the Institut Jules Bordet, Brussels, Belgium (40 patients enrolled). Eligible patients were enrolled consecutively in Lausanne; in Brussels, consecutive eligible patients were randomized 1:4 to this study or to an ongoing therapeutic study of the European Organization for Research and Treatment of Cancer (EORTC) International Antimicrobial Therapy Cooperative Group (IATCG), respectively.

\section{Antibiotic treatment}

Two grams of cefepime (Bristol-Myers Squibb Co., Princeton, NJ, USA) were administered three times daily. The antibiotic was reconstituted with sterile water for infusion, mixed with between 50 and $100 \mathrm{~mL}$ of sodium chloride $0.9 \%$ and infused i.v. over a 30 -min period. Cefepime was given for a minimum of 7 days or until resolution of all signs and symptoms of infection, except in patients with a non-infectious fever in whom therapy was discontinued after 4 days.

\section{Classification of febrile episode}

According to the definitions of the IATCG of the EORTC, primary febrile episodes were classified as microbiologically documented infections with or without bacteraemia, clinically documented infections, unexplained fever (formerly designated as possible infections) or non-infectious fever (i.e. fever associated with the underlying 
neoplasia or related to chemotherapy or to the transfusion of blood products) (EORTC, 1991 a). A bacteraemia was defined as the isolation of a pathogenic microorganism from one or more blood culture bottles. For coagulase-negative staphylococci, corynebacteria or other skin contaminants, two sets of positive blood cultures were required, unless the same organism was isolated concomitantly from another infected site.

\section{Evaluation of response}

The response to cefepime therapy was evaluated according to the definitions of the IATCG of the EORTC (EORTC, 1991a). These results were compared with those obtained using the definitions of Pizzo et al. (1986) and those of the Immunocompromised Host Society (IHS) (1990).

The definitions used to assess the response to empirical therapy with cefepime in evaluable patients were as follows:

(1) IATCG of the EORTC. Success was resolution of fever and all clinical signs of infections (whenever present) and eradication of the infecting microorganism (whenever isolated) without discontinuation of cefepime or addition of other antibacterial agents; response must have been maintained for at least 4 days after discontinuation of cefepime. Failure was either: death from primary infection; persistence of bacteraemia beyond the first $24 \mathrm{~h}$ of therapy; breakthrough bacteraemia; or no response to therapy (defined as the persistence of a pathogen and/or fever in a patient whose condition was not improving and who required discontinuation of cefepime or the addition of other antibacterial agents). The addition of antiviral or antifungal agents without a concomitant change in the antibacterial therapy was not considered a failure.

(2) Criteria of Pizzo et al. (1986). Success without modification referred to episodes in which the patient successfully recovered from fever and neutropenia without the need for the addition of antimicrobial agents or the modification of the initial, randomly assigned regimen. Success with modification referred to episodes in which the patient successfully recovered from the fever and neutropenia but required a modification of the assigned regimen. Failure referred to death resulting from a documented or presumed infection during the neutropenic episode.

(3) IHS. Success: all signs, symptoms, and microbiological evidence of infection had resolved with the primary therapy. No recurrence of infection was found for at least 1 week after discontinuation of the initial antibacterial regimen. Initial response but regimen modified (IRBRM): the initial pathogen was eradicated by the primary regimen but a second infection/fever developed which fell outside the spectrum of the primary antibacterial(s) and which required the addition of another antimicrobial agent (e.g. antifungal, antiviral or antiparasitic). Failure: the addition to, modification of, or change in the initial antibacterial regimen in order to eradicate the primary infection resulted in the initial regimen being designated as having failed. Death due to infection would also be classified as a failure. Non-response (applicable only to patients with unexplained fever): the addition of a non-antibacterial antibiotic (e.g. antifungal, antiviral or antiparasitic) for a specific indication or of an antibacterial agent because of a new bacterial infection in a patient with unexplained fever who had not responed (i.e. defervesced) to the initial antibacterial regimen caused the episode to be designated a non-response. 


\section{Further infections and death}

Further infections were defined as new infections caused by an organism not recognized as the initial pathogen and which occurred either during therapy or within I week after discontinuation of therapy. Death was attributed to infection when it occurred as a direct consequence of either the presenting or a further infection.

\section{Toxicity}

Hypokalaemia was defined as a fall in the serum potassium of $\geqslant 0.5 \mu \mathrm{mol} / \mathrm{L}$ in the absence of gastrointestinal loss or treatment with another potassium-depleting drug. Nephrotoxicity was defined as a rise in the serum creatinine of $>45 \mu \mathrm{mol} / \mathrm{L}$ if the baseline creatinine was $\leqslant 260 \mu \mathrm{mol} / \mathrm{L}$ or a rise of $>90 \mu \mathrm{mol} / \mathrm{L}$ if the baseline creatinine was $>260 \mu \mathrm{mol} / \mathrm{L}$ in the absence of other causes of renal dysfunction (e.g. hypotension or hypovolaemia) or other potentially nephrotoxic drugs (e.g. cisplatin, amphotericin B, acyclovir or vancomycin). Hepatotoxicity was defined as a two-fold increase in bilirubin or serum aspartate aminotransferase and serum alanine aminotransferase in the absence of other potentially hepatotoxic drugs or any other causes of hepatic dysfunction.

\section{Microbiology}

Standardized identification and antibiotic susceptibility testing were used (Barry \& Thornsberry, 1991; Isenberg et al., 1991; Sahm \& Washington, 1991). Susceptibility data were evaluated according to the zone diameter interpretative standards and the equivalent MICs recommended by the National Committee for Clinical Laboratory Standards (NCCLS, 1990a,b). An isolate was considered resistant to cefepime if the MIC was $\geqslant 32 \mathrm{mg} / \mathrm{L}$ or if the inhibitory zone diameter by the Kirby-Bauer method was $\leqslant 15 \mathrm{~mm}$ (data on file Bristol-Myers Squibb Co., Princeton, NJ, USA).

\section{Results}

Between January 1990 and May 1991, 108 febrile episodes in 84 granulocytopenic cancer patients were entered into the trial. Fifteen patients who together accounted for 36 episodes of fever were included on multiple occasions, all during separate episodes of neutropenia. For the remainder of this paper, the terms 'episodes' and 'patients' will be used interchangeably.

Seventeen patients could not be evaluated for response to therapy for the following reasons: a protocol violation in six patients (unjustified discontinuation of cefepime in four and non-eligibility in two); a fever of non-infectious origin in six patients (fever was due to cancer in five patients and to the infusion of granulocyte-macrophage colony-stimulating factor (GM-CSF) in one); a non-bacterial infection in four patients (herpes simplex stomatitis in three and pulmonary tuberculosis in one); and the discontinuation of therapy because of an anaphylactic reaction in one patient.

Of the 91 evaluable patients, there were 48 males and 43 females with a mean age of 48 years (range 17-78). The underlying conditions were haematological malignancies in 57 patients $(63 \%)$ and solid tumours in 34 patients $(37 \%)$. Seven patients $(8 \%)$ had undergone allogeneic bone marrow transplantation. 
At study entry, patients had a median granulocyte count of $0.3 \times 10^{y} / \mathrm{L}$ (range $0-0.992 \times 10^{9} / \mathrm{L}$ ). Profound granulocytopenia (i.e. $\leqslant 0.1 \times 10^{9} / \mathrm{L}$ ) was present in $64 \%$ of patients. At the time of entry, patients had been granulocytopenic for a median of 6.5 days (range $0-65$ ). The median duration of granulocytopenia while receiving therapy was 8 days (range 1-35). Overall, $62 \%$ of the patient-trial days were spent with granulocyte counts of $\leqslant 0.1 \times 10^{9} / \mathrm{L}, 19 \%$ with counts between 0.101 and $0.5 \times 10^{9}, 7 \%$ with counts between 0.501 and $1.0 \times 10^{9} / \mathrm{L}$ and $12 \%$ with counts $>1.0 \times 10^{9} / \mathrm{L}$.

Fifty-three patients $(58 \%)$ had received oral antibacterial prophylaxis which comprised a fluoroquinolone in 50 patients (pefloxacin $400 \mathrm{mg}$ bd in 36 , norfloxacin $400 \mathrm{mg}$ bd in 13 and ciprofloxacin $250 \mathrm{mg}$ bd in one); this was administered in combination with penicillin $\mathrm{V}(500 \mathrm{mg}$ bd) in ten patients. Erythromycin, spiramycin and co-trimoxazole were given to one patient each. Oral antifungal prophylaxis was given to 51 patients $(56 \%)$ and included amphotericin B ( $100 \mathrm{mg} 6$ hourly) in 31 cases, amphotericin B (500 mg qds) in seven, fluconazole ( $200 \mathrm{mg}$ od) in four and itraconazole (200 $\mathrm{mg}$ bd) in nine.

Details of the 91 evaluable febrile episodes are shown in Table I. Microbiologically documented infections occurred in 25 patients $(27 \%)$. Eighteen infections $(72 \%)$ were caused by Gram-positive bacteria and seven $(28 \%)$ by Gram-negative bacteria. Bacteraemia was detected in 18 patients; 15 of the episodes were caused by Gram-positive bacteria. Fourteen of $18(78 \%)$ Gram-positive pathogens and all of the Gram-negative pathogens were susceptible to cefepime. The four Gram-positive

Table I. Documentation of infection in the 91 evaluable episodes

\begin{tabular}{lc}
\hline Type of infection & No. of episodes \\
\hline Microbiologically documented infections & $25(27 \%)$ \\
with bacteraemia & 18 \\
without bacteraemia & 7 \\
Gram-positive bacterial pathogen & 18 \\
coagulase-negative staphylococci & 8 \\
streptococcl & 5 \\
viridans streptococci & 3 \\
$\beta$-haemolytic streptococci & \multicolumn{2}{|c}{1} \\
S. pneumoniae & \multicolumn{1}{c}{1} \\
Staphylococcus aureus & 3 \\
other organisms & \\
Gram-negative bacterial pathogens & 2 \\
Pseudomonas aeruginosa & 7 \\
Escherichia coli & 2 \\
other & 2 \\
Clinically documented infections & 3 \\
sites of infection & $47(52 \%)$ \\
oral cavity and pharynx & \\
lower respiratory tract & 28 \\
skin and soft tissues & 10 \\
gastrointestinal tract & 7 \\
Unexplained fever & 2 \\
\hline ane Clostium septiam
\end{tabular}

${ }^{a}$ One Clostridium septicum isolate and one Stomatococcus mucilaginosus isolate.

${ }^{h}$ One isolate each of Proteus vulgarts, a Leptotrichia sp. and Klebstella pneumoniae. 
Table II. Influence of antibacterial prophylaxis on the development of infection

\begin{tabular}{lcc}
\hline Classification of infection & $\begin{array}{c}\text { Administered } \\
\text { prophylaxis }\end{array}$ & $\begin{array}{c}\text { Not administered } \\
\text { prophylaxis }\end{array}$ \\
\hline $\begin{array}{l}\text { Number of patients } \\
\text { Microbiologically documented infection }\end{array}$ & 53 & $38^{a}$ \\
$\quad \begin{array}{l}\text { Gram-positive infection } \\
\text { Gram-negative infection }\end{array}$ & $10(19 \%)$ & $8(21 \%)^{b}$ \\
Clinically documented infection & $1(2 \%)$ & $6(16 \%)^{c}$ \\
Unexplained fever & $32(60 \%)$ & $15(39 \%)^{d}$ \\
\hline
\end{tabular}

Fisher's exact test (two-tailed): ${ }^{a} P=0.05 ;{ }^{h} P=0.05 ;{ }^{\prime} P=0.02 ;{ }^{d} P=0.06 ;{ }^{\circ} P=0.61$.

bacteria resistant to cefepime included three coagulase-negative staphylococci causing catheter-related infections and one isolate of Clostridium septicum which was responsible for a fatal episode of septic shock. Only one of these four bacteria (a coagulasenegative staphylococcus) was isolated from a patient who had previously been treated with cefepime. The sites of these microbiologically documented infections were the skin and soft tissues (ten patients, three of whom had catheter-related infections), the oral cavity or upper respiratory tract (five patients), the lower respiratory tract (three patients) and the gastrointestinal tract (one patient). In six patients the primary site of infection could not be identified. Clinically documented infection occurred in 47 patients $(52 \%)$; the most frequently implicated sites of infection were the oral cavity and pharynx $(60 \%)$. In 19 patients the cause of fever was unexplained.

As shown in Table II, the use of antibacterial prophylaxis had a significant impact on the documentation of infection $(P=0.05)$. Gram-negative infections occurred more often in patients who had not received antibacterial prophylaxis $(16 \%$ vs $2 \%, P=$ 0.02 ), whereas clinically documented infections were more common in patients who had received prophylaxis $(60 \%$ vs $39 \%, P=0.06)$. Of the 11 microbiologically documented infections occurring in patients who received prophylaxis with fluoroquinolones, ten were bacteraemias, eight of which were caused by organisms (three coagulase-negative staphylococci, three viridans streptococci and one isolate each of Stomatococcus mucilaginosus and a Leptotrichia sp.) resistant to the antibiotic used for prophylaxis.

The response rates of each category of infection to cefepime monotherapy are shown in Table III. Response was evaluated according to the criteria of the IATCG of the EORTC and the results were compared with those obtained with the other two methods of assessment. Overall, $71 \%$ of infectious episodes responded to cefepime alone. For microbiologically documented infections, clinically documented infections and unexplained fevers, response rates were $56 \%, 77 \%$ and $79 \%$ respectively. Six of seven $(86 \%)$ Gram-negative infections, but only eight of $18(44 \%)$ Gram-positive infections, resolved with cefepime monotherapy. The response rate for Gram-positive infections rose to $57 \%(8 / 14)$ when only Gram-positive bacteria which were susceptible in vitro to cefepime were considered.

Of the ten Gram-positive infectious episodes which failed to respond, there were three deaths which were attributed to the primary infections. Two patients died of septic shock $24 \mathrm{~h}$ after the initiation of therapy; the pathogens were a cefepime-susceptible strain of $S$. aureus and a cefepime-resistant Clostridium septicum isolate. The third patient died on the seventh day of treatment from irreversible acute respiratory distress syndrome (ARDS) associated with Streptococcus pneumoniae bacteraemia (susceptible 


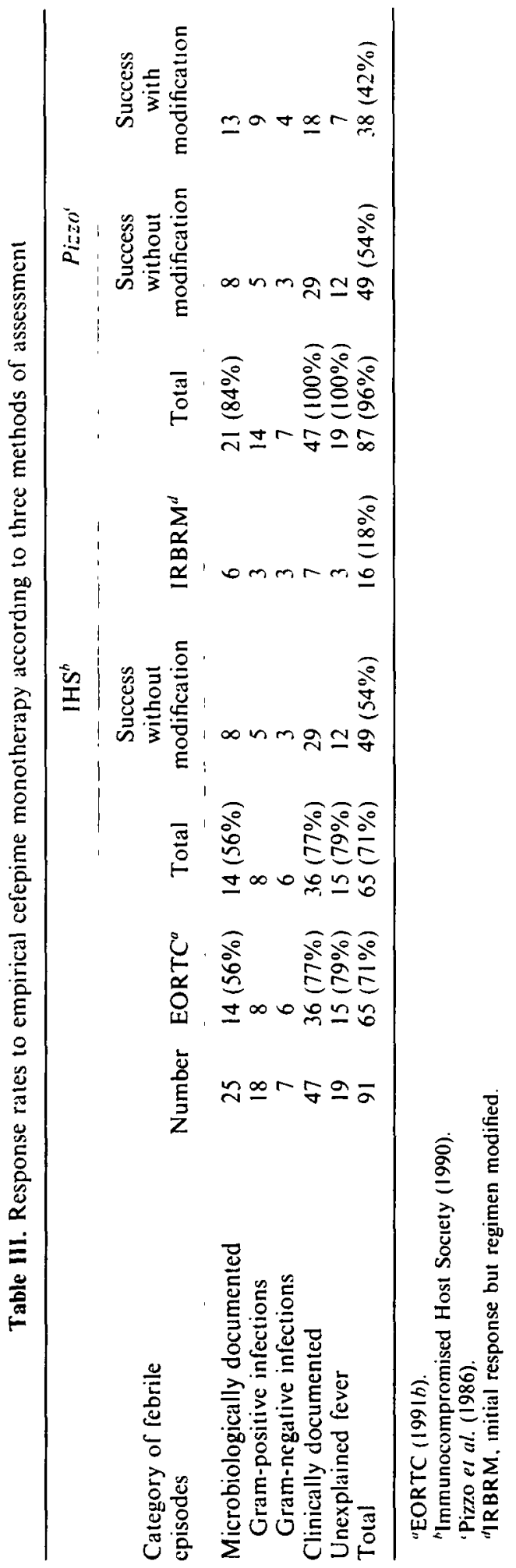


to cefepime). Although this patient's granulocytopenia had resolved after only two days of treatment, he remained febrile until his death. In two patients, failure was due to persistence of fever and the development of ARDS 3 and 4 days after documentation of bacteraemia caused by a cefepime-susceptible strain of Streptococcus mitis. Five failures occurred in patients with coagulase-negative staphylococcal catheter infections. Bacteraemia persisted during therapy in three patients, two of whom were infected with cefepime-resistant strains. Another patient failed to respond because of non-bacteraemic infection caused by a cefepime-resistant bacterium. In the last patient, fever persisted until modification of therapy and catheter removal. The only failure in patients with Gram-negative infection was a catheter-related bacteraemic episode caused by a cefepime-susceptible Leptotrichia sp. which was cured after removal of the catheter and modification of therapy.

Thirty-six of $47(77 \%)$ of the clinically documented infections were cured with cefepime monotherapy. The reasons for failure included exacerbation of the infection (five patients) or the persistence of fever and infection (six patients). Therapy was modified after a mean of 6 days. In four patients with unexplained fever, antibiotic therapy was changed after a mean of 6.5 days because the patient remained febrile.

When the data were analysed according to the guidelines of the Immunocompromised Host Society, the response rates for each category of infection were identical to those obtained with the EORTC criteria. Yet, 16 of 65 responders $(25 \%)$ developed secondary infections (see further infections below) which required modifications of therapy. Therefore, these patients were classified as IRBRM. Modifications of therapy included the administration of another antibacterial agent to 12 patients (additional to cefepime in six patients), acyclovir to two patients and amphotericin $B$ to one.

If success of therapy was defined as the survival of a patient who was free of infection after resolution of granulocytopenia (see Pizzo's criteria), the overall response rate was $96 \%$ ( 87 of 91 patients). Response rates were $84 \%$ for microbiologically documented infections and $100 \%$ for both clinically documented infections and unexplained fevers (Table III). Forty-nine patients $(54 \%)$ improved with cefepime monotherapy and remained afebrile until resolution of granulocytopenia (success without modification). Thirty-eight patients (42\%) improved with modification of therapy (success with modification) which cured either the primary infection (22 episodes) or secondary infections ( 16 episodes) which developed in patients who had responded to the initial cefepime monotherapy.

Secondary infections developed in 16 patients $(18 \%)$. Of these, 13 were microbiologically documented and included seven episodes of septicaemia caused by Candida albicans (two episodes), Streptococcus sanguis (one), Streptococcus salivarius (one), Enterococcus faecalis (one), P. aeruginosa (one) and Bacteroides fragilis (one); only the $S$. sanguis and $S$. salivarius isolates were susceptible to cefepime. The non-bacteraemic infections included Clostridium difficile colitis (three cases which occurred 5, 7 and 12 days respectively after initiation of cefepime therapy), $S$. aureus osteomyelitis (one case), Aspergillus fumigatus pneumonia (one) and herpetic stomatitis (one). Proctitis with ulceration and two episodes of unexplained fever accounted for the remaining secondary infections. All but one of these infections occurred during episodes of profound ( $<0.1 \times 10^{9} / \mathrm{L}$ ) and persistent (mean 15 days; range 6-38) granulocytopenia, mostly in patients with leukaemia. With the exception of one case of disseminated candidiasis, all secondary infections were cured with appropriate therapy. 
All 108 patients who were entered into the study were evaluated for safety. Abnormalities of liver function tests were noted in 13 patients $(12 \%)$, of whom six had received other potentially hepatotoxic agents. In all cases, liver dysfunction was mild (the median increase in bilirubin or serum glutamic-oxaloacetic transaminase/serum glutamic-pyruvic transaminase (SGOT/SGPT) concentrations was $2 \cdot 3$-fold, with a range of 0.5 - to 6-fold), did not require premature discontinuation of therapy and was rapidly and fully reversible. Diarrhoea developed in four patients $(4 \%)$. A minor rash developed in six patients $(6.5 \%)$ after 2 to 10 days of treatment. Nausea related to the infusion of cefepime was noted in two patients. One patient developed generalized urticaria after the first dose of cefepime; this was the only instance in which therapy was discontinued prematurely because of toxicity.

\section{Discussion}

In this open study, we evaluated the efficacy of empirical monotherapy with cefepime, a new advanced-generation cephalosporin with extended-spectrum activity against methicillin-susceptible staphylococci and streptococci. As reported in many other recent clinical trials, Gram-positive bacteria, particularly staphylococci and streptococci, were the predominant pathogens (Cohen et al., 1983; EORTC, 1991a; Novakova et al., 1991; Winston et al., 1991; Cornelissen et al., 1992; Rolston et al., 1992). Of note, oral prophylaxis with fluoroquinolones had a significant impact on the development of infection. All but one of the Gram-negative infections occurred in patients who had not received prophylaxis with these agents $(P=0.05)$. In contrast, clinically documented infections predominated in patients who had received fluoroquinolones. Whether oral prophylaxis with fluoroquinolones causes a shift from microbiologically to clinically documented infections or whether it actually reduces the proportion of febrile episodes in these patients is an unresolved issue.

The results of the present study showed that monotherapy with cefepime is effective; overall, the response rate with cefepime alone was $71 \%$. In addition, $26 \%$ of primary infections improved after modification of treatment. These results are comparable with those obtained with ceftazidime and imipenem-cilastatin monotherapy (Sanders, Powe \& Moore, 1991; Winston et al., 1991; Rolston et al., 1992). The response rates were excellent for Gram-negative infections, clinically documented infections and fevers of unexplained origin. On the other hand, only $44 \%$ of patients with Gram-positive infections responded to cefepime monotherapy, although an additional $45 \%$ responded with modification of therapy. In view of the excellent in-vitro activity of cefepime against Gram-positive bacteria, one might have expected a better response rate for infections caused by these organisms. However. close analysis of the ten Gram-positive failures revealed that unfavourable clinical conditions or in-vitro resistance, rather than a lack of clinical efficacy, accounted for most of these. Three patients died of Gram-positive infections; two of these were in septic shock when cefepime treatment was started and died within $24 \mathrm{~h}$. It is unlikely that any antibiotic would have improved the outcome of patients with such fulminant and often intractable infection. The third patient died on the seventh day of treatment with irreversible ARDS and $S$. pneumoniae bacteraemia. The pneumococcus was highly susceptible to cefepime and the patient's granulocytopenia had already recovered. Five patients failed therapy because of catheter-related, coagulase-negative staphylococcal infection. Three of the strains were methicillin-resistant and vancomycin therapy was required. In the other 
two patients, bacteraemia or fever persisted with cefepime therapy; the catheter was therefore removed and treatment modified. Subsequent improvement was related more to removal of the catheter than to modification of therapy. In two patients with cefepime-susceptible $S$. mitis bacteraemia, cefepime therapy was discontinued after 3 and 4 days respectively because of the development of ARDS. Similar cases, where adequate therapy failed to prevent the onset of ARDS, have been reported by others (EORTC, 1991 a,b; Elting, Bodey \& Keefe, 1992).

In order to assess both the true response to empirical monotherapy with cefepime, as well as all subsequent events occurring throughout the period of granulocytopenia, data were analysed according to three different sets of criteria. Identical results were obtained with both the EORTC and IHS criteria. Both of these methods focused on the evaluation of the initial response to empirical therapy. According to the IHS criteria, responders were classified into two subgroups: those who remained afebrile until granulocytopenia had resolved $(54 \%)$ and those who subsequently developed a secondary infection and who required a modification of empirical therapy (i.e. initial response but regimen modified) (18\%). Analysis of the results according to the criteria used by Pizzo et al. (1986) showed a survival rate of $96 \%$ after resolution of granulocytopenia. Success of therapy (i.e. survival of the patient) was interpreted in the light of whether or not the empirical therapy was modified. Although this method of assessment allowed us to evaluate the responses for both early (primary) and late (secondary) infections, the exact response rate to cefepime monotherapy could not, however, be derived directly from the data. Indeed, relying on only 'success without modification' to estimate the true response rate would have led to an underestimation, because 16 responders who later developed secondary infections and who required modifications of therapy (defined as IRBM according to the IHS criteria) were put into the category 'success with modification'. Assessment of the current data according to three different sets of criteria emphasizes the need to present results in such a way that enables the efficacy of empirical therapy, the overall response rate and patient survival after the resolution of granulocytopenia to be evaluated.

In summary, in this open pilot study, cefepime monotherapy was shown to be effective and safe as empirical treatment of fever in cancer patients with granulocytopenia. Whether cefepime is superior to other advanced-generation cephalosporins for the treatment of infections caused by methicillin-susceptible staphylococci or streptococci will require evaluation in large. prospective comparative studies.

\section{References}

Barry, A. L. \& Thornsberry, C. (1991). Susceptibility tests: diffusion test procedures. In Manual of Clinical Microbiology (Balows, A., Hausler, W. J., Hermann, K. L., Isenberg, H. D. \& Shadomy, H. J., Eds), pp. 1117-25. American Society for Microbiology, Washington, DC.

Calandra, T. \& Cometta, A. (1991). Antibiotic therapy for Gram-negative bacteremia. Infectious Disease Clinics of North America 5, 817-34.

Cohen, J., Donelly, J. P., Worsley, A. M., Catovsky, D., Goldman, J. M. \& Galton, D. A. G. (1983). Septicemia caused by vindans streptococci in neutropenic patients with leukaemia. Lancet ii, 1452-4.

Cornelissen. J. J.. de Graeff, A., Verdonck, L. F., Branger. T., Rozenberg-Arska, M., Verhoef, J. et al. (1992). Imipenem versus gentamicin combined with either cefuroxime or cephalothin as initial therapy for febrile neutropenic patients. Antimicrobial Agents and Chemotherapy $36,801-7$. 
Deresinski, S., de Pauw, B. E. \& Feld, R. (1991). Empirical therapy of Gram-negative bacteremia in granulocytopenic patients with leukemia. In Program and Abstracts of the Thirty-First Interscience Conference on Antimicrobial Agents and Chemotherapy, Atlanta, GA, 1991. p. 128. American Society for Microbiology, Washington, DC.

Dornbusch, K., Mortsell, E. \& Goransson, E. (1990). In vitro activity of cefepime, a new parenteral cephalosporin, against recent European blood isolates in comparison with piperacillin/tazobactam. Chemotherapy 36, 259-67.

Elting, L. S., Bodey, G. P. \& Keefe, B. H. (1992). Septicemia and shock syndrome due to viridans streptococci: a case-control study of predisposing factors. Clinical Infectious Diseases 14, $1201-7$.

EORTC International Antimicrobial Therapy Cooperative Group. (1987). Ceftazidime combined with a short or long course of amikacin for empirical therapy of Gram-negative bacteremia in cancer patients with granulocytopenia. New England Journal of Medicine 317, 1692-8.

EORTC International Antimicrobial Therapy Cooperative Group. (1991a). Single versus multiple daily doses of amikacin combined with ceftriaxone or ceftazidime for the empirical therapy of fever in granulocytopenic cancer patients. In Program and Abstracts of the Thirty-First Interscience Conference on Antimicrobial Agents and Chemotherapy, Atlanta, $G A, 1991$. p. 128. American Society for Microbiology, Washington, DC.

EORTC International Antimicrobial Therapy Project Group. (1991b). Vancomycin added to empirical combination antibiotic therapy for fever in granulocytopenic cancer patients. Journal of Infectious Diseases 163, 951-8.

Fannstein, V., Bodey, G. P., Elting, L., Bolivar, E. R., Keating, M. J., McCreadie, K. B. et al. (1983). A randomized study of ceftazidime compared to ceftazidime and tobramycin for the treatment of infections in cancer patients. Journal of Antimicrobial Chemotherapy 12, Suppl. $A, 101-10$.

Immunocompromised Host Society. (1990). The design, analysis, and reporting of clinical trials on the empirical antibiotic management of the neutropenic patient. Journal of Infectious Diseases 161, 397-401.

Isenberg, H. D., Washington, J. A., Doern, G. V. \& Amsterdam, D. (1991). Specimen collection and handling. In Manual of Clinical Microbiology (Balows, A., Hausler, W. J., Hermann, K. L. \& Isenberg, H. D., Eds), pp. 2-15. American Society for Microbiology, Washington, DC.

Kessler, R. E., Bies, M., Buck, R. E., Chisholm, D. R., Pursiano, T. A., Tsai, Y. H. et al. (1985). Comparison of a new cephalosporin, BMY-28142, with other broad-spectrum $\beta$-lactam antibiotics. Antimicrobial Agents and Chemotherapy 27, 207-16.

National Committee for Clinical Laboratory Standards. (1990a). Performance Standards for Antimicrobial Disk Susceptibility Tests. Approved Standard M2-A4. NCCLS, Villanova, PA.

National Committee for Clinical Laboratory Standards. (1990b). Methods for Dilution Antimicrobial Susceptibility Tests for Bacteria That Grow Aerobically. Approved Standard $M 7-A 42$. NCCLS, Villanova, PA.

Novakova, I., Donnelly, J. P. \& De Pauw, B. (1991). Ceftazidime as monotherapy or combined with teicoplanin for initial empiric treatment of presumed bacteremia in febrile granulocytopenic patients. Antimicrobial Agents and Chemotherapy 35, 672-8.

Oster, S., Edelstein, H., Cassanbo, K. \& McCabe, R. (1990). Open trial of cefepime (BMY-28142) for infections in hospitalized patients. Chemotherapy 34, 954-7.

Pizzo, P. A., Hathorn, J. W., Hiemenz, J., Browne, M., Commers, J. \& Cotton, D. (1986). A randomized trial comparing ceftazidime alone with combination antibiotic therapy in cancer patients with fever and neutropenia. New England Journal of Medicine 315, 552-8.

Ramphal, R., Bolger, M., Oblon, D. J., Sherertz, R. J., Malone, J. D., Rand, K. H. et al. (1992). Vancomycin is not an essential component of the initial empiric treatment regimen for febrile neutropenic patients receiving ceftazidime: a randomized prospective study. Antimicrobial Agents and Chemotherapy 36, 1062-7.

Rolston, K. V. I., Berkey, P., Bodey, G. P., Anaissie, E. J., Khardori, N. M., Joshi, J. H. et al. (1992). A comparison of imipenem to ceftazidime with or without amikacin as empiric therapy in febrile neutropenic patients. Archives of Internal Medicine 152, 283-91. 
Rubin, M., Hathorn, J. W., Marshall. D., Gress. J.. Steinberg. S. M. \& Pizzo. P. A. (1988). Gram-positive infections and the use of vancomycin in 550 episodes of fever and neutropenia. Annals of Internal Medicine 108, 30-5.

Sahm, D. F. \& Washington, J. A. (1991). Antibacterial susceptibility tests: dilution methods. In Manual of Clinical Microbiology (Balows. A.. Hausler. W. J.. Hermann. K. L.. Isenberg, H. D. \& Shadomy, H. J.. Eds). pp. 1105-16. American Society for Microbiology. Washington, DC.

Sanders, J. W., Powe, N. R. \& Moore. R. D (1991). Ceftazidime monotherapy for empiric treatment of febrile neutropenic patients: a meta-analysis. Journal of Infectious Diseases 164, 907-16

Winston, D. J., Ho, W. G.. Bruckner, D. A. \& Champlin, R. E. (1991). $\beta$-Lactam antibiotic therapy in febrile granulocytopenic patients. A randomized trial comparing cefoperazone plus piperacillin. ceftazidime plus piperacillin. and imipenem alone. Annals of Internal Medicine 115, 849-59.

Young, L. S. (1989). Neutropena: antibiotic combinations for empiric therapy European Journal of Microbiology and Infectious Diseases 8, 118-22. 